\title{
Cognitive Dysfunction Relates to Subjective Report of Mental Fatigue in Patients with Chronic Fatigue Syndrome
}

\author{
Lucile Capuron', Leonie Welberg', Christine Heim', Dieter Wagner', Laura Solomon ${ }^{2,4}$, \\ Dimitris A Papanicolaou ${ }^{3,5}$, R Cameron Craddock ${ }^{2}$, Andrew H Miller' and William C Reeves*,2 \\ 'Department of Psychiatry and Behavioral Sciences, Emory University School of Medicine, Atlanta, GA, USA; ${ }^{2}$ Division of Viral and Rickettsial \\ Diseases, National Center for Infectious Diseases, Centers for Disease Control and Prevention, Atlanta, GA, USA; ${ }^{3}$ Department of Medicine, \\ Emory University School of Medicine, Atlanta, GA, USA
}

\begin{abstract}
Patients with chronic fatigue syndrome (CFS) frequently complain of cognitive dysfunction. However, evidence of cognitive impairment in CFS patients has been found in some, but not other, studies. This heterogeneity in findings may stem from the relative presence of mental fatigue in the patient populations examined. The present study assessed this possibility in a population-based sample of CFS patients. In all, 43 patients with CFS defined by the criteria of the 1994 research case definition using measurements recommended by the 2003 International CFS Study Group, and 53 age-, sex-, and race/ethnicity-matched nonfatigued subjects were included in the study. Mental fatigue was assessed using the mental fatigue subscale of the multidimensional fatigue inventory. Cognitive function was evaluated using an automated battery of computerized tests (Cambridge neuropsychological test automated battery (CANTAB)) that assessed psychomotor function, planning and problem-solving abilities, and memory and attentional performance. CFS patients with significant complaints of mental fatigue (score of mental fatigue 2 standard deviations above the mean of nonfatigued subjects) exhibited significant impairment in the spatial working memory and sustained attention (rapid visual information processing) tasks when compared to CFS patients with low complaints of mental fatigue and nonfatigued subjects. In CFS patients with significant mental fatigue, sustained attention performance was impaired only in the final stages of the test, indicating greater cognitive fatigability in these patients. CFS patients with low mental fatigue displayed performance comparable to nonfatigued subjects on all tests of the CANTAB battery. These findings show strong concordance between subjective complaints of mental fatigue and objective measurement of cognitive impairment in CFS patients and suggest that mental fatigue is an important component of CFS-related cognitive dysfunction.
\end{abstract}

Neuropsychopharmacology (2006) 3 I, 1777- 1784. doi:I 0. I038/sj.npp. I 30 1005; published online 4 January 2006

Keywords: chronic fatigue syndrome; cognition; mental fatigue; working memory; CANTAB

\section{INTRODUCTION}

Chronic fatigue syndrome (CFS) is a debilitating disorder that has been particularly challenging for health-care providers to evaluate and treat. There are no characteristic physical signs or laboratory abnormalities. Rather, CFS is defined as profound physical or mental fatigue of at least 6 -months duration, that is not relieved by rest, causes

\footnotetext{
*Correspondence: Dr WC Reeves, Division of Viral and Rickettsial Diseases, National Center for Infectious Diseases, Centers for Disease Control and Prevention, 1600 Clifton Road, Mail Stop AI5, Atlanta, GA 30333, USA, Tel: + I 404639 0221, E-mail: wcrl@cdc.gov ${ }^{4}$ Current address: Human Research Protection Office, Office of the Chief Science Officer, Centers for Disease Control and Prevention, Atlanta, GA 30333, USA

${ }^{5}$ Current address: Merck \& Co., Inc., Rahway, NJ 07065, USA

Received 10 August 2005; revised and accepted 8 November 2005 Online publication: 10 November 2005 at http://www.acnp.org/citations/ Npp I | | 005050502/default.pdf
}

substantial reduction in previous levels of occupational, educational, social, or personal activities, cannot be explained by concurrent medical or psychiatric disease, and is accompanied by at least four of eight case-defining symptoms: unusual post-exertional (mental or physical) fatigue, impaired memory or concentration, unrefreshing sleep, headaches, muscle pain, joint pain, sore throat, and tender cervical nodes (Fukuda et al, 1994). CFS is a complex, multidimensional illness in which fatigue can manifest in multiple forms, including physical fatigue and mental fatigue. Indeed, it may very well be that the differential manifestations of symptom complexes within the CFS syndrome may account for the variability that often characterizes the disorder. Ultimately, it has been proposed that an operational CFS case definition will need to be based on empirical studies designed to examine the possibility that distinct biological pathways may result in the specific symptomatology of CFS (Reeves et al, 2003).

One important symptom domain in CFS is cognitive dysfunction. Complaints of cognitive dysfunction are 
frequent in CFS patients. Studies have reported that 50-85\% of patients with CFS report cognitive difficulties and that these contribute considerably to their social and occupational dysfunction (Christodoulou et al, 1998; Komaroff and Buchwald, 1991; Michiels and Cluydts, 2001). Neurocognitive dysfunction in patients with CFS manifests primarily in the form of concentration/attention problems, memory impairment, poor word-finding ability, decreased information-processing speed, motor slowing, and mental exhaustion (Busichio et al, 2004; Deluca et al, 2004; Michiels and Cluydts, 2001). Cognitive dysfunction in CFS patients has been found in some, but not all, objective studies (Afari and Buchwald, 2003; Michiels and Cluydts, 2001; Ross et al, 2004; Wearden and Appleby, 1997). This heterogeneity may stem from the relative presence of mental fatigue in the patients with CFS. To further assess this possibility, the present study evaluated the relationship between subjective complaints of mental fatigue and cognitive function using a validated automated neuropsychological battery (Cambridge neuropsychological test automated battery (CANTAB)) in a population-based sample of CFS patients.

\section{METHODS}

\section{Subjects}

This study adhered to human experimentation guidelines of the Helsinki Declaration and was approved by the CDC and Emory University Institutional Review Boards. All subjects gave informed consent.

The present study enrolled subjects who had participated in the 1997-2000 Wichita population-based CFS Surveillance Study (Reyes et al, 2003). In brief, the surveillance study followed a cohort of 7162 fatigued and nonfatigued adults representative of the Wichita population at 12-, 24-, and 36-month intervals, with telephone interviews and clinical evaluations. Participants in the current study were a subset of the 659 fatigued adults ever clinically evaluated during surveillance study and who ever met the criteria for CFS. Upon admission to this study, subjects were re-evaluated in terms of CFS symptoms and exclusionary medical and psychiatric conditions. The 43 subjects (mean age $(S D)=50$ (9) years, 7 men, 36 women) who met the 1994 criteria (Fukuda et al, 1994) for current CFS comprise the cases in this report. In all, 53 age-, sex-, race/ethnicitymatched nonfatigued subjects (mean age $(\mathrm{SD})=50$ (9) years, 10 men, 43 women) were randomly selected from the cohort, who participated in surveillance at the baseline and all follow-up periods, who did not have medical or psychiatric exclusions, and who had not reported fatigue of at least 1-month duration. Similarly to the nonfatigued subject, none of the CFS patients included in the present study had any medical or psychiatric exclusions or suffered from current major depressive disorder (as assessed during a structured clinical interview for DSM-IV (SCID)) at the time of the evaluation. As current classification as CFS was not completely concurrent with recruitment classification (time at which demographic matching was done), strict matching was not maintained (eg, some patients initially recruited were not anymore 'current' CFS at the time of the study), but cases and controls remained nevertheless demographically comparable.

\section{Assessment and Classification of CFS}

Subjects who agreed to participate were admitted to a Wichita hospital research unit for 2 days. To identify exclusionary medical conditions (Fukuda et al, 1994; Reeves et al, 2003), they provided a standardized past medical history, a review of current medications, underwent a standardized physical examination, and provided blood and urine for routine analysis, including a complete blood count and blood and urine chemistries.

Subjects were classified as CFS if they met the criteria of the 1994 case definition (Fukuda et al, 1994) and followed the recommendations of the International CFS Study Group (Reeves et al, 2003) concerning measurement of the major illness domains. The Medical Outcomes Survey Short Form-36 (SF-36) was used to measure functional impairment (Ware and Sherbourne, 1992), the multidimensional fatigue inventory (MFI) to evaluate severity of fatigue (Smets et al, 1995), and the CDC symptom inventory to document the occurrence, duration, and severity of accompanying symptoms (Wagner et al, 2005). Substantial reduction in occupational, educational, social, or recreational activities was defined as scores lower than the 25th percentile on the physical function $(\leqslant 70)$, or role physical $(\leqslant 50)$, or social function $(\leqslant 75)$, or role emotional $(\leqslant 66.7)$ subscales of SF-36. Severe fatigue was defined as $\geqslant$ medians of the MFI general fatigue $(\geqslant 13)$ or reduced activity $(\geqslant 10)$ scales. Finally, the presence of substantial accompanying symptoms was defined by $\geqslant 4$ symptoms and scoring $\geqslant 25$ on the Symptom Inventory Case Definition Subscale. Subjects who met all three criteria (SF-36, MFI, and symptom inventory) when they entered the clinical study were classified as CFS $(n=43)$. Subjects with no evidence of fatigue documented by the MFI were classified as nonfatigued.

\section{Assessment of Mental Fatigue}

Mental fatigue was assessed by the MFI mental fatigue subscale, which was not used to classify subjects as CFS. Four items contribute to the mental fatigue subscale ('When I am doing something, I can keep my thoughts on it', 'I can concentrate well', 'It takes a lot of efforts to concentrate on things', 'My thoughts easily wander') on a 5-point scale, with 1 corresponding to the absence of fatigue and 5 to severe fatigue. Accordingly, the total score of mental fatigue ranges from 4 (best) to 20 (worst).

\section{Neuropsychological Assessments}

Cognitive function was assessed with the CANTAB (Robbins and Sahakian, 1994), a battery of neuropsychological tests on a touch screen portable computer. This ensures a standardized and homogenous form of testing with instantly available data and detailed recording of the subject's accuracy and speed of responses. Seven tests of the CANTAB battery designed to assess specific cognitive functions were used.

Psychomotor coordination and motor speed. The reaction time (RTI) test was used to evaluate psychomotor retardation, assumed to be associated with basal ganglia dysfunction. 
The test includes simple and five-choice RTI tasks and is divided into five stages, requiring increasingly complex chains of responses and providing distinction between reaction (or decision) time and movement latencies.

Reasoning and planning abilities. The Stockings of Cambridge (SOC) task was used to evaluate spatial planning/ problem solving. The task makes substantial demands on executive function and is sensitive to frontal-lobe deficits (Robbins et al, 1998). The test is based on the Tower of London test and contains incremental levels of difficulty. The subject was shown two displays containing colored balls, and had to use the balls in the lower display to copy the pattern shown in the upper one. The subject was told not to make the first move until he/she was confident that he/she could execute the entire sequence needed to solve the problem. The time taken to copy the pattern (including the time taken to make the first move after the presentation of the display (initial thinking time (ITT)) and the time spent thinking about a problem during its execution (subsequent thinking time (STT))), the number of perfect solutions (problems solved in a minimum of moves), and the average number of moves required to solve the problem were taken as the subject's planning abilities. The copying task was followed by a following task controlling for motor performance, in which the upper display moved one ball at a time, repeating the moves made by the subject in the corresponding previous planning phase.

Memory. Three tests of memory were employed. (a) The spatial working memory (SWM) is a test of self-ordered searching task sensitive to fronto-subcortical dysfunction (Owen et al, 1995). The test requires that subjects find blue tokens in a series of displayed boxes and use the tokens to fill up an empty column, while not returning to boxes where a blue token had been previously found. The number of boxes increased from three to eight. The number of between-search errors (errors made when the subject revisits a box in which a token has previously been found) and a strategy score derived from the number of search sequences in the four, six, and eight boxes were used as performance indexes. The strategy score retraced the 'route' previously used by subjects in searching through the spatial array of boxes (Owen et al, 1992; Robbins, 1996). One possible strategy was to follow a predetermined sequence, beginning with a particular box and then returning to start each new sequence with the same box as soon as a token was found. The lower the strategy score, the more efficient was the subject. This test has previously been reported to be impaired in CFS patients (Joyce et al, 1996). (b) The pattern recognition memory (PRM) test assesses visual recognition memory in a two-choice forced discrimination paradigm and is sensitive to temporal or hippocampal dysfunctions. Percent correct responses (successful recognition) and response latency for correct responses were used as performance indices. (c) The spatial recognition memory (SRM) test is a test of spatial recognition in a two-choice forced discrimination paradigm. As for PRM, percent correct responses (successful recognition) and response latency for correct responses were used as performance indices for SRM.
Attention. Two tests were used to evaluate attention: (a) The attentional shift: intra/extra dimensional shift (IED) task is a test of rule acquisition and reversal, featuring visual discrimination and attentional set shifting analogous to the Wisconsin card sorting test (WCST). This test is sensitive to cognitive dysfunction in Parkinson's disease, basal ganglia lesions, and frontal-lobe deficits (Owen et al, 1991; Swainson and Robbins, 2001). Two stimuli (one correct, one incorrect) in the form of shapes and lines are presented in four possible locations on the computer screen. First, the subject has to maintain attention to different examples of stimuli within the same dimension (shapes), while distracting stimuli of different dimensions are present (lines) (intradimensional shifts). Second, the subject has to shift attention to the previously irrelevant dimension (lines) and ignore the previously relevant dimension (shapes) (extra dimensional shifts). Subjects progress through the test by satisfying a set of criteria of learning at each stage (nine stages in total). If at any stage the subject fails to reach this criterion after 50 trials, the test ends. The total number of stages achieved, the total number of errors (adjusted to the number of completed stages), the errors made up to the extra dimensional shift (pre-ED errors), as well as the errors at extra dimensional shift (EDS errors) served as performance indices. (b) The rapid visual information processing (RVIP) test is a 4-min visual continuous performance (sustained attention/vigilance) task with a small working memory component. Digits (ranging from 2 to 9 ) appeared one at a time (100 digits/min) in the center of the computer screen in a random order. Subjects were asked to press a response pad when they detected any one of three number sequences ('2-4-6', '4-68 ', and ' $3-5-7$ '). The performance on the RVIP test was measured using the metric $A^{\prime}$ (a signal detection measure of sensitivity to errors regardless of error tendency that assesses the global performance on the test using both the probability of hits and the probability of false alarms, and ranging from 0.00 to 1.00 ; bad to good), the mean latencies for correct responses, the total number of hits, and total number of false alarms. In addition, cognitive fatigability in the RVIP test was assessed for each subject's comparing the performance (response latencies) during the first period of the test (trials $1-9$, min 2) to the performance during the second (trials $10-$ 18 , min 3) and third periods of the test (trials 19-27, min 4).

The mean duration for the execution of the neurocognitive battery (including the instructions) was approximately $1 \mathrm{~h}$. The order in which tests were presented was counterbalanced for each patient. Of note, CANTAB tests are culture- and language-free, and there are no gender-related differences in performance (Robbins and Sahakian, 1994).

\section{Data Analysis}

Mental fatigue scores on the MFI were compared between CFS patients and nonfatigued subjects using a two-sample $t$-test. Subsequent analyses of cognitive performance were performed contrasting CFS patients with significant mental fatigue $v s$ CFS patients with low mental fatigue and nonfatigued subjects. Mental fatigue was considered to be significant if the score of mental fatigue was 2 standard deviations above the mean mental fatigue score in nonfatigued subjects. Analysis of variance (ANOVA) was used to compare performance across the three groups in the 
selected CANTAB tests. Post-hoc comparisons were performed when appropriate using both conservative (Tukey) and powerful ( $t$-test) tests of significance. When significance was attained using both tests, only the Tukey test was reported. Analyses including a time/period factor (eg, effects of test period on performance on the RVIP task) were performed using a two-way repeated-measure ANOVA with group as the between-subject factor and time as the repeated-measure factor. All probabilities were two-sided and the degree of significance was set at $P<0.05$.

\section{RESULTS}

Subjects with CFS exhibited significantly greater MFI mental fatigue scores (mean: 14.1 (SD: 4.2)) than nonfatigued subjects (mean: 6 (SD: 2$)$ ) $(T=12.5, P<0.0001)$ (Figure 1). Out of the 43 CFS patients, 34 (79\%) reported significant mental fatigue (as defined by a score of mental fatigue above the mean ( $+2 \mathrm{SD})$ mental fatigue score in nonfatigued subjects, ie, score $>10$ ). The remaining nine CFS patients had a low mental fatigue (score of mental fatigue $\leqslant 10$ ). All nonfatigued controls had mental fatigue scores $\leqslant 10$. Apart from the dimensions of mental fatigue and general fatigue (mental fatigue: $T=-8.63, P<0.001$; general fatigue: $T=-2.98, P<0.01)$, scores on all other dimensions of fatigue assessed by the MFI scale, including physical fatigue, reduced activity, and reduced motivation, were not statistically different between CFS patients with significant mental fatigue and CFS patients with low mental fatigue $(T=-1.33 ; T=-1.68 ; T=-1.35 ;$ all $P>0.05)$. In addition, there was no difference in terms of age (50 vs 53 years old,), sex, and psychotropic medication (antidepressants, anxiolytics, sleeping pills, stimulants) use between CFS patients with significant mental fatigue and CFS patients with low mental fatigue (age: $50 v s 53, T=-1.12$; $P=0.49$; gender (male/female): $5 / 29$ vs $2 / 7, \chi^{2}(1)=0.29$; $P=0.59 ;$ psychotropic medication use: 59 vs $67 \%$, $\left.\chi^{2}(1)=0.18 ; P=0.67\right)$. Of note, both CFS groups exhibited greater psychotropic medication use than nonfatigued controls $(15 \%)$. To further assess the influence of mental fatigue on cognitive function in CFS patients, analyses of

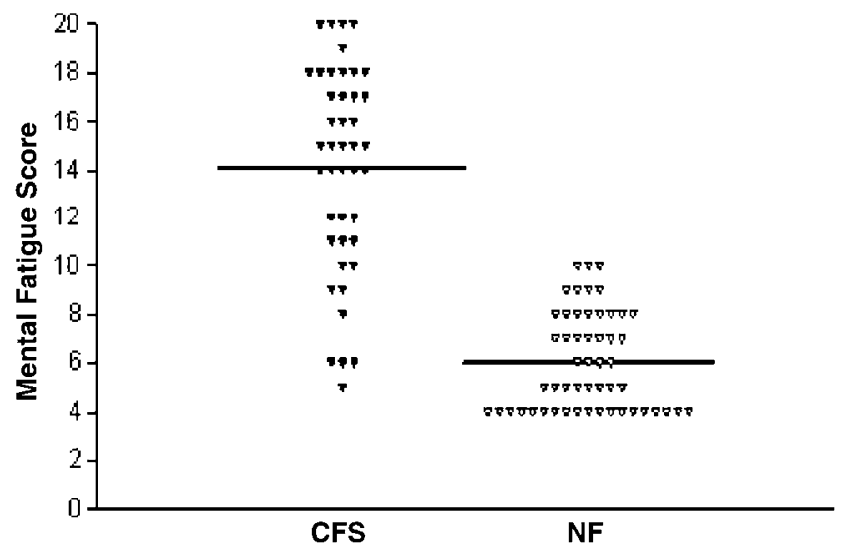

Figure I Scores on the subscale of mental fatigue of the MFI (Smets et al, 1995) in patients with CFS $(\boldsymbol{\nabla}, N=43)$ and nonfatigued subjects (NF, $\nabla, N=53)$. The horizontal black lines represent the mean score in each group. cognitive performance were conducted distinguishing CFS patients with significant mental fatigue (score $>10)$ from CFS patients with low mental fatigue (score $\leqslant 10)$.

\section{Psychomotor Coordination and Motor Speed}

As depicted in Table 1, there was no difference between groups in terms of psychomotor coordination and motor speed, as indicated by performance on the RTI test. Simple choice reaction and movement times as well as five-choice reaction and movement time were comparable between groups (ANOVA: $\mathrm{F}(2,93)=0.43 ; \mathrm{F}(2,93)=0.95 ; \mathrm{F}(2,93)=$ 1.13; $\mathrm{F}(2,93)=2.79$, respectively, all $P>0.05)$.

\section{Reasoning and Planning Abilities}

Reasoning and planning abilities (as assessed by the SOC test) were comparable across groups (Table 2). All groups exhibited similar performance in terms of the mean number

Table I Psychomotor Coordination and Motor Speed

\begin{tabular}{|c|c|c|c|}
\hline & $\begin{array}{l}\text { CFS+MF } \\
(N=34)\end{array}$ & $\begin{array}{c}\text { CFS-MF } \\
(N=9)\end{array}$ & $\begin{array}{c}\text { NF } \\
(N=53)\end{array}$ \\
\hline \multicolumn{4}{|l|}{ Reaction time test } \\
\hline Simple reaction time (ms) & $373(11)$ & $375(28)$ & $361(8)$ \\
\hline Simple movement time (ms) & $506(17)$ & $548(45)$ & $493(16)$ \\
\hline Five-choice reaction time (ms) & $399(9)$ & $429(29)$ & $397(8)$ \\
\hline Five-choice movement time (ms) & $502(15)$ & $546(54)$ & $466(14)$ \\
\hline
\end{tabular}

Data are shown as mean (SE).

CFS+MF: CFS patients with significant mental fatigue (mental fatigue score $>10$ ); CFS-MF: CFS patients with low mental fatigue (mental fatigue score $\leqslant 10)$; NF: nonfatigued subjects.

Table 2 Reasoning and Planning Abilities

\begin{tabular}{lrrr}
\hline & $\begin{array}{c}\text { CFS+MF } \\
(\mathbf{N}=\mathbf{3 4})\end{array}$ & $\begin{array}{c}\text { CFS-MF } \\
(\mathbf{N}=\mathbf{9})\end{array}$ & $\begin{array}{c}\mathbf{N F} \\
(\mathbf{N}=\mathbf{5 3})\end{array}$ \\
\hline $\begin{array}{l}\text { Stockings of Cambridge } \\
\text { Total \# perfect solutions }\end{array}$ & $8.7(0.3)$ & $8.2(0.3)$ & $8.9(0.3)$ \\
& & & \\
Four-move problems & & & \\
Average \# moves & $5.4(0.2)$ & $5.6(0.4)$ & $5.3(0.1)$ \\
ITT & $11.4(1.2)$ & $9.4(2.4)$ & $11.6(1.2)$ \\
STT & $2.8(0.5)$ & $2.0(0.6)$ & $1.9(0.3)$ \\
& & & \\
Five-move problems & & & \\
Average \# moves & $6.6(0.2)$ & $7.1(0.5)$ & $6.4(0.2)$ \\
ITT & $13.9(2.5)$ & $8.3(1.4)$ & $13.7(1.4)$ \\
STT & $1.3(0.2)$ & $2.5(1.5)$ & $1.0(0.1)$
\end{tabular}

Data are shown as mean (SE).

CFS+MF: CFS patients with significant mental fatigue (mental fatigue score $>$ I0); CFS-MF: CFS patients with low mental fatigue (mental fatigue score $\leqslant 10)$; NF: nonfatigued subjects.

ITT: initial thinking time; STT: subsequent thinking time. 
of moves, ITT, and STT for both four-move problems and five-move problems (ANOVA (four-move problems) moves: $\mathrm{F}(2,93)=0.27$; ITT: $\mathrm{F}(2,93)=0.30$; STT: $\mathrm{F}(2,93)=$ 1.37 , all $P>0.05$; ANOVA (five-move problems) - moves: $\mathrm{F}(2,93)=1.31$; ITT: $\mathrm{F}(2,93)=0.91 ;$ STT: $\mathrm{F}(2,93)=3.07$, all $P>0.05)$. Similarly, there were no significant differences between groups in the total number of problems solved in a minimum number of moves (ANOVA: $\mathrm{F}(2,93)=0.50$, $P>0.05)$

\section{Memory}

CFS patients with significant mental fatigue exhibited impairment in memory, notably in SWM, in comparison with CFS patients with low mental fatigue and nonfatigued subjects (ANOVA: $\mathrm{F}(2,93)=2.23, P=0.014$ ) (Table 3). More specifically, patients with CFS and significant mental fatigue scores (CFS + MF) displayed significantly impaired search strategy (as revealed by a higher strategy score in the SWM test) compared to nonfatigued subjects $(P=0.03)$ and CFS patients with low mental fatigue $(\mathrm{CFS}-\mathrm{MF})(P=0.05)$. In terms of between-search errors, ANOVA failed to indicate a significant difference between the three groups at the designed statistical threshold $(P<0.05)$ (ANOVA: $\mathrm{F}(2,93)=$ 2.23, $P=0.11)$. Nevertheless, complementary analysis indicated that CFS patients with significant mental fatigue made significantly more between-search errors during the SWM test compared with nonfatigued subjects ( $t$-test; $P=0.04$ ) (Figures $2 \mathrm{a}$ and $2 \mathrm{~b}$ ). Working memory performance of CFS patients with low mental fatigue was not statistically different from performance in nonfatigued subjects.

Performance accuracy was similar in the three groups on the PRM and SRM tests (ANOVA: $\mathrm{F}(2,93)=0.73$ and $\mathrm{F}(2,93)=0.82$, all $P>0.05)$. Nevertheless, for response latencies in the SRM, there was a trend for a significant difference between groups (ANOVA: $\mathrm{F}(2,93)=2.65$, $P=0.08)$. Complementary analysis indicated that CFS

Table 3 Performance on Tests of Memory

\begin{tabular}{lllc}
\hline & $\begin{array}{c}\text { CFS+MF } \\
(\mathbf{N}=\mathbf{3 4})\end{array}$ & $\begin{array}{c}\text { CFS-MF } \\
(\mathbf{N}=\mathbf{9})\end{array}$ & $\begin{array}{c}\text { NF } \\
(\mathbf{N}=\mathbf{5 3})\end{array}$ \\
\hline $\begin{array}{l}\text { Spatial working memory } \\
\quad \text { Strategy score }\end{array}$ & $35.7(0.6)^{*} \boldsymbol{*}^{\dagger}$ & $32.2(2.0)$ & $33.5(0.5)$ \\
Between-search errors & $34.6(2.8)^{\dagger}$ & $29.8(5.6)$ & $26.4(2.5)$ \\
& & & \\
Pattern recognition memory & & & \\
\% Correct & $89.6(1.5)$ & $94.0(2.2)$ & $89.9(1.5)$ \\
$\quad$ Mean correct latency (s) & $2.24(0.12)$ & $2.08(0.23)$ & $2.20(0.11)$ \\
& & & \\
Spatial recognition memory & & & \\
\% Correct & $83.8(1.6)$ & $81.1(2.6)$ & $84.7(1.0)$ \\
Mean correct latency (s) & $2.80(0.18)^{\dagger}$ & $2.99(0.55)$ & $2.40(0.10)$ \\
\hline
\end{tabular}

Data are shown as mean (SE)

CFS+MF: CFS patients with significant mental fatigue (mental fatigue score $>$ 10); CFS-MF: CFS patients with low mental fatigue (mental fatigue score

$\leqslant$ I0); NF: nonfatigued subjects. ${ }^{*} P=0.05$ vs CFS $-M F$; ${ }^{\dagger} P<0.05$ vs NF. patients with significant mental fatigue exhibited significantly longer response latencies in the SRM test compared with nonfatigued subjects $(t$-test; $P<0.05)$. In the PRM test, response latencies were similar across groups (ANOVA: $\mathrm{F}(2,93)=1.18, P>0.05)$.

\section{Attention}

The ability to shift attentional set (as assessed by the IED test) was comparable across the three groups (Table 4). Indeed, no difference was found between CFS patients with significant complaints of mental fatigue, CFS patients with low mental fatigue and nonfatigued subjects in the number of completed stages, the number of total errors, as well as the number of shift errors (EDS errors) and pre-shift errors (pre-ED errors) in the IED test (ANOVA - stages: $\mathrm{F}(2,93)=0.67$; total errors: $\mathrm{F}(2,93)=0.94$; $\mathrm{EDS}$ errors: $\mathrm{F}(2,91)=0.48$; Pre-ED errors: $\mathrm{F}(2,93)=1.74$; all $P>0.05)$.

Similarly, there was no significant overall difference in the global performance of the three groups on the RVIP test, as measured by signal $A^{\prime}$, mean latencies to correct responses,
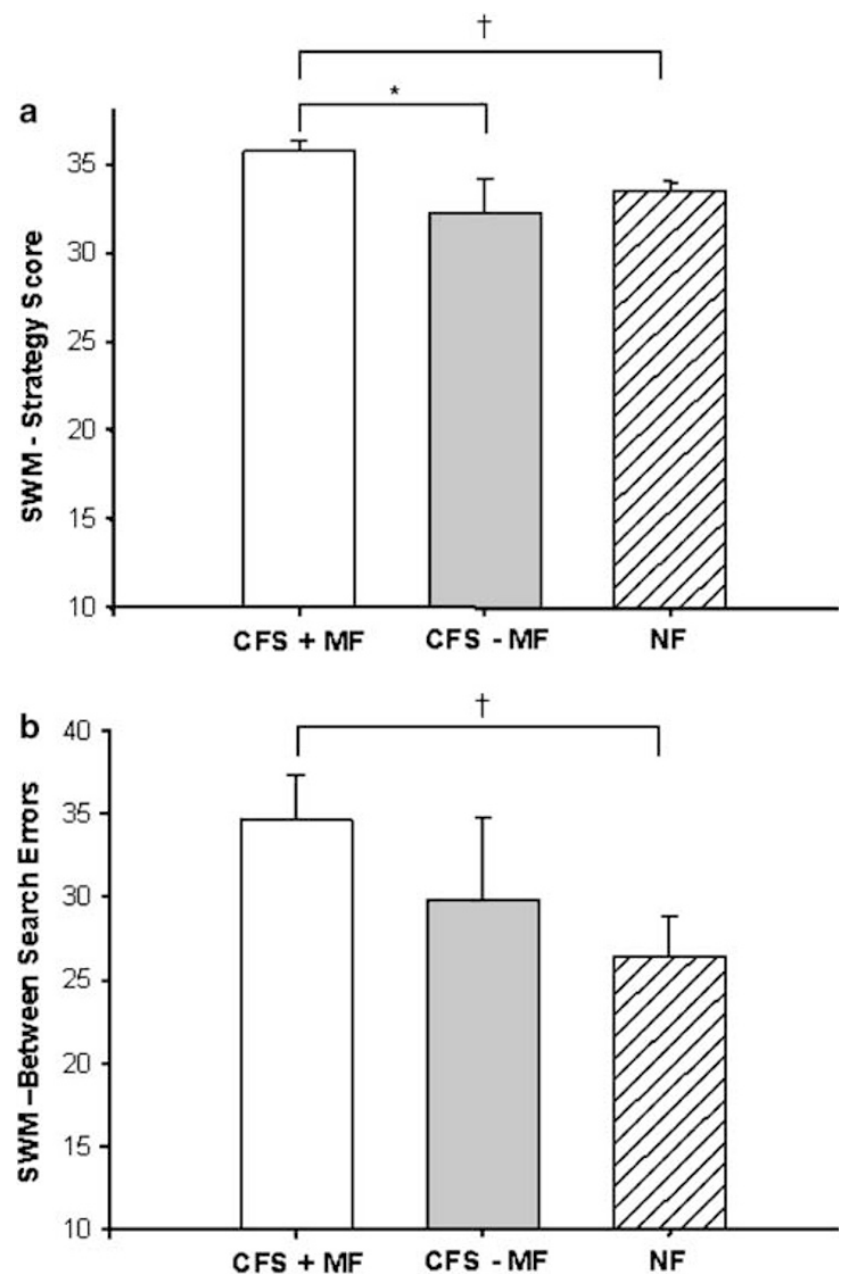

Figure 2 Strategy score (a) and between-search errors (b) in patients with CFS and significant complaints of mental fatigue (CFS + MF), patients with chronic fatigue and low mental fatigue (CFS-MF), and nonfatigued subjects (NF). The lower the strategy score the more efficient was the subject. $* P=0.05 \mathrm{CFS}+\mathrm{MF}$ vs CFS-MF; ${ }^{\dagger} P<0.05 \mathrm{CFS}+\mathrm{MF}$ vs NF. 
Table 4 Performance on Tests of Attention

\begin{tabular}{|c|c|c|c|}
\hline & $\begin{array}{l}\text { CFS+MF } \\
(N=34)\end{array}$ & $\begin{array}{c}\text { CFS-MF } \\
(N=9)\end{array}$ & $\begin{array}{c}\text { NF } \\
(N=53)\end{array}$ \\
\hline \multicolumn{4}{|l|}{ ID/ED shift } \\
\hline Stages completed & $8.6(0.1)$ & $8.2(0.3)$ & $8.4(0.2)$ \\
\hline Total errors (adjusted) & $24.2(3.4)$ & $34.9(7.1)$ & $30.4(3.9)$ \\
\hline EDS errors ${ }^{\mathrm{a}}$ & $10.2(1.8)$ & |3.8 (3.9) & $10.2(1.4)$ \\
\hline Pre-EDS errors & $6.9(0.7)$ & $7.1(0.9)$ & $9.3(1.0)$ \\
\hline \multicolumn{4}{|c|}{ Rapid visual information processing } \\
\hline$A^{\prime b}$ & $0.89(0.01)$ & $0.89(0.01)$ & $0.91(0.01)$ \\
\hline Mean latency $(\mathrm{ms})^{\mathrm{b}}$ & $532(20)$ & $518(23)$ & $526(15)$ \\
\hline Total false alarms & $1.9(1.2)$ & $0.9(0.4)$ & $0.9(0.2)$ \\
\hline Total hits & $14.7(1.0)^{\dagger}$ & $15.4(1.5)$ & $17.2(0.7)$ \\
\hline
\end{tabular}

Data are shown as mean (SE).

CFS+MF: CFS patients with significant mental fatigue (mental fatigue score $>$ 10); CFS-MF: CFS patients with low mental fatigue (mental fatigue score $\leqslant$ (0); NF: nonfatigued subjects. ${ }^{\dagger} P<0.05$ vs NF.

a Data for this parameter were unavailable for two nonfatigued subjects who did not reach the stage of extra-dimensional shift.

${ }^{b}$ One CFS subject with significant mental fatigue missed all target sequences, and therefore did not obtain any values for these measurements.

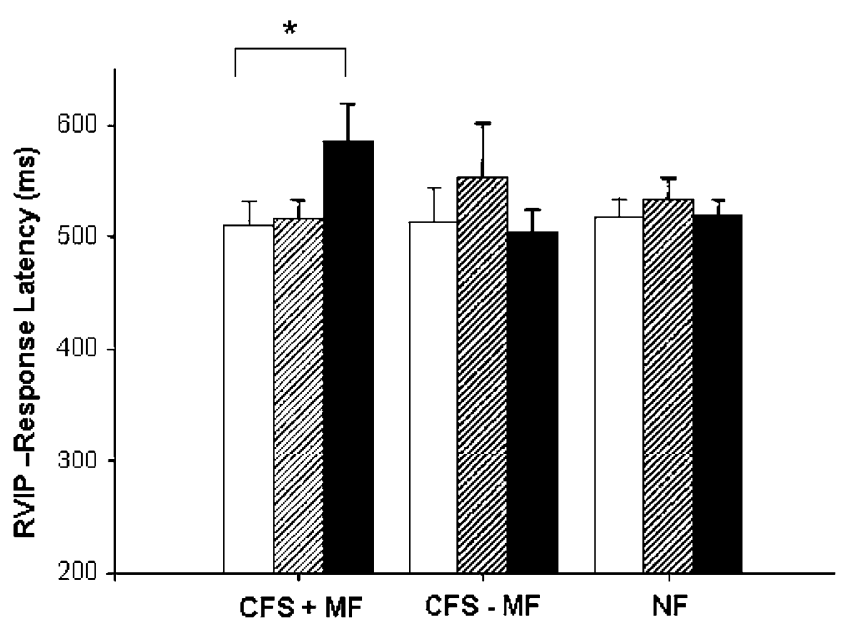

Figure 3 Change in response latency $(\mathrm{ms})$ over the course of the RVIP test in patients with CFS and significant complaints of mental fatigue (CFS + MF), patients with chronic fatigue and low mental fatigue (CFS-MF), and nonfatigued subjects (NF). White bar: first period of the test (trials I-9, ie, min 2); striped bar: second period of the test (trials 10-18, ie, min 3); solid black bar: third period of the test (trials 19-27, ie, min 4). Only CFS patients with significant complaints of mental fatigue exhibited increased response latencies between the first and last periods of the test (mean increase $=78 \mathrm{~ms}$ ). $* P<0.05$ period I vs period 3 .

total false alarms, and total hits (ANOVA: $A^{\prime} \mathrm{F}(2,92)=1.90$; mean latency $\mathrm{F}(2,92)=0.06$; false alarms $\mathrm{F}(2,93)=0.54$; hits $\mathrm{F}(2,93)=2.18$; all $P>0.05)$. Nevertheless, complementary analysis ( $t$-test) indicated that the total number of hits in the RVIP test was significantly lower in CFS patients with significant mental fatigue compared with nonfatigued subjects $(P=0.04)$. A two-way repeated-measure ANOVA with group as a between-subject factor and RVIP trial period (trials 1-9 vs trials 10-18 vs trials 19-27) was performed to compare changes in response latencies (as a measure of cognitive fatigability) during the course of the RVIP test in the three groups. Results did not show any significant main effect of group or trial period on the evolution of response latencies during the course of the RVIP test $(\mathrm{F}(2,91)=0.18$ and $\mathrm{F}(2,182)=1.17$, respectively, all $P>0.05$ ), but revealed a significant group $\times$ trial period interaction $(\mathrm{F}(4,182)=3.19, P=0.014)$. Examination of the simple main effects and post-hoc analyses revealed that changes in response latencies over the course of the RVIP test were apparent only in CFS patients with significant mental fatigue. In those patients, response latencies were significantly increased in the final stages of the test (trials 19-27) in comparison with earlier trials, suggesting cognitive fatigability in these patients $(P=0.03)$ (Figure 3 ). Similar to nonfatigued subjects, CFS patients with no significant complaints of mental fatigue did not show any significant impairment in the RVIP task.

\section{DISCUSSION}

The present findings indicate that mental fatigue is an important correlate of cognitive impairment in CFS patients. To our knowledge, this is the first time that a clear association is demonstrated between subjective complaints of mental fatigue and objective measurements of cognitive function in a population-based sample of patients with CFS. In addition, these data provide important information regarding the specificity of cognitive dysfunction in patients with CFS. Finally, these findings provide interesting clues regarding the neural pathways that may underlie cognitive alterations in CFS patients.

As noted previously, cognitive dysfunction has been found in some, but not all, neuropsychological studies in CFS patients. For instance, whereas several investigations have shown evidence of impaired memory, attention, and information-processing speed in patients with CFS (Deluca et al, 2004, 1997; Dobbs et al, 2001; Joyce et al, 1996), other reports have failed to show any significant cognitive alterations in these patients (Michiels and Cluydts, 2001). This discrepancy between results may be due to the variability of methods of assessment of cognitive function, but also may be due to the relative presence of mental fatigue or other relevant symptoms in the patient populations examined. In support of the latter hypothesis, ratings of subjective mental fatigue as assessed by the MFI were found to significantly relate to cognitive performance, especially in tests of information-processing speed, verbal fluency, and retention, in patients with myasthenia gravis (Paul et al, 2002).

In the present study, patients with CFS and significant complaints of mental fatigue (as indicated by a score of mental fatigue on the MFI scale superior to the mean +2 SD of the mental fatigue score in nonfatigued subjects) were found to exhibit significant impairment in working memory, especially in the form of impaired search strategy and increased number of between-search errors. This finding is in accordance with previous reports indicating alterations in working memory, including the SWM task of the CANTAB, in patients with CFS (Joyce et al, 1996; Marshall et al, 1997). In addition, CFS patients with 
significant complaints of mental fatigue showed significant impairment in the task of sustained attention/vigilance, as revealed by increased response latencies to continuous stimuli, notably at the final stages of the RVIP task, suggesting higher cognitive fatigability in these patients. This finding is supported by previous data indicating reduced information-processing speed in CFS patients (Marshall et al, 1997, 1999). CFS patients with significant mental fatigue did not show, however, any significant slowing in tasks requiring lower cognitive demand/control, including the task of simple and choice RTI and PRM. Attentional shift abilities, as well as planning and problemsolving performance, were not impaired in patients with CFS with significant complaints of mental fatigue compared to CFS patients with low mental fatigue and nonfatigued subjects. Nevertheless, it should be noted that, because of the limited sample size, especially for the group of CFS patients with significant mental fatigue, it may also be that the absence of impairment in these tests of executive function in CFS patients with mental fatigue is related to reduced statistical power. Interestingly, patients with CFS and low mental fatigue exhibited performance on all tasks (including the SWM and the RVIP tasks found to be impaired in CFS patients with significant mental fatigue) similar to nonfatigued subjects. In addition to demonstrating a strong concordance between subjective complaints of mental fatigue and objective measurement of cognitive function in CFS patients, these findings indicate that mental fatigue represents an essential determinant of cognitive dysfunction associated with CFS and a relevant variable to be considered when assessing cognitive performance in patients with this disorder. Of note, cognitive impairment in CFS patients with mental fatigue was not attributable to mood disorders or psychotropic medication use in these patients.

The tasks that were found to be impaired in CFS patients with mental fatigue (ie, SWM and RVIP tasks) have been shown to involve specific fronto-parieto-thalamo-striatal areas. More specifically, the task of spatial working memory was found to rely primarily on the dorsolateral prefrontal (DLPFC) cortex (Beats et al, 1996), and performance on the rapid visual information-processing task has been shown to be associated with increased activity in prefrontal and parietal regions, thalamus, caudate, anterior insula, middle occipital/fusiform gyrus, and cerebellar areas (Lawrence et al, 2002). Taken together, these findings suggest that changes in neural circuits involving the frontal and parietal cortex as well as the thalamus and basal ganglia may constitute a primary pathway in the pathophysiology of CFS-related mental fatigue and cognitive dysfunction. Nevertheless, it remains also possible that CFS patients recruit different or additional brain areas than those detected in normal controls to perform the same tasks. This hypothesis is supported by recent data indicating that CFS patients engaged in a complex auditory informationprocessing task utilize more extensive regions of the network associated with verbal WM system than nonfatigued subjects (Lange et al, 2005). Finally, given the heterogeneity and diversity of symptoms in CFS, it is also highly probable that not one, but several, pathophysiological mechanisms are involved in the syndrome of chronic fatigue. Consistent with this notion, data obtained in patients with Parkinson disease afflicted with fatigue indicate that mental fatigue and physical fatigue (as assessed by the MFI scale) are independent symptoms, suggesting that these two forms of fatigue involve separate etiologies and pathophysiological pathways (Lou et al, 2001). Accordingly, whereas dopamine agents appeared to be useful for improving physical fatigue (Lou et al, 2003), drugs targeting serotonin and/or noradrenergic function may be more adapted for the treatment/alleviation of mental fatigue and cognitive dysfunction. In support of this hypothesis, the antidepressant paroxetine, a serotonin reuptake inhibitor, was found to prevent mental fatigue and cognitive dysfunction, but not physical fatigue, in patients with medical illness affected with fatigue and treated with the cytokine, interferon-alpha (Capuron et al, 2002).

In conclusion, our findings show a clear relationship between perceived mental fatigue and objective measures of working memory and cognitive fatigability in CFS patients, and indicate that complaints of mental fatigue constitute an important factor to be considered in the detection and assessment of cognitive dysfunction in patients with CFS. It appears, therefore, quite possible that the lack of consideration (or the underestimation) of complaints of mental fatigue in CFS patients may have contributed to the heterogeneity of results obtained from the assessment of neuropsychological performance in patients with fatiguing illnesses.

\section{FUNDING}

This study was funded by the Centers for Disease Control and Prevention. The findings and conclusions in this report are those of the authors and do not necessarily represent the views of the funding agency.

\section{REFERENCES}

Afari N, Buchwald D (2003). Chronic fatigue syndrome: a review. Am J Psychiatry 160: 221-236.

Beats BC, Sahakian BJ, Levy R (1996). Cognitive performance in tests sensitive to frontal lobe dysfunction in the elderly depressed. Psychol Med 26: 591-603.

Busichio K, Tiersky LA, Deluca J, Natelson BH (2004). Neuropsychological deficits in patients with chronic fatigue syndrome. $J$ Int Neuropsychol Soc 278-285.

Capuron L, Gumnick JF, Musselman DL, Lawson DH, Reemsnyder A, Nemeroff CB et al (2002). Neurobehavioral effects of interferon-alpha in cancer patients: phenomenology and paroxetine responsiveness of symptom dimensions. Neuropsychopharmacology 26: 643-652.

Christodoulou C, DeLuca J, Lange G, Johnson SK, Sisto SA, Korn L et al (1998). Relation between neuropsychological impairment and functional disability in patients with chronic fatigue syndrome. J Neurol Neurosurg Psychiatry 64: 431-434.

Deluca J, Christodoulou C, Diamond BJ, Rosenstein ED, Kramer N, Natelson BH (2004). Working memory deficits in chronic fatigue syndrome: differentiating between speed and accuracy of information processing. J Int Neuropsychol Soc 10: 101-109.

Deluca J, Johnson SK, Ellis SP, Natelson BH (1997). Cognitive functioning is impaired in patients with chronic fatigue syndrome devoid of psychiatric disease. J Neurol Neurosurg Psychiatry 62: 151-155.

Dobbs BM, Dobbs AR, Kiss I (2001). Working memory deficits associated with chronic fatigue syndrome. J Int Neuropsychol Soc 7: 285-293. 
Fukuda K, Straus SE, Hickie I, Sharpe MC, Dobbins JG, Komaroff A (1994). The chronic fatigue syndrome: a comprehensive approach to its definition and study. International Chronic Fatigue Syndrome Study Group. Ann Intern Med 121: 953-959.

Joyce E, Blumenthal S, Wessely S (1996). Memory, attention, and executive function in chronic fatigue syndrome. $J$ Neurol Neurosurg Psychiatry 60: 495-503.

Komaroff AL, Buchwald D (1991). Symptoms and signs of chronic fatigue syndrome. Rev Infect Dis 13(Suppl 1): S8-S11.

Lange G, Steffener J, Cook DB, Bly BM, Christodoulou C, Liu WC et al (2005). Objective evidence of cognitive complaints in chronic fatigue syndrome: a BOLD fMRI study of verbal working memory. Neuroimage 26: 513-524.

Lawrence NS, Ross TJ, Stein EA (2002). Cognitive mechanisms of nicotine on visual attention. Neuron 36: 539-548.

Lou JS, Kearns G, Benice T, Oken B, Sexton G, Nutt J (2003). Levodopa improves physical fatigue in Parkinson's disease: a double-blind, placebo-controlled, crossover study. Mov Disord 18: 1108-1114.

Lou JS, Kearns G, Oken B, Sexton G, Nutt J (2001). Exacerbated physical fatigue and mental fatigue in Parkinson's disease. Mov Disord 16: 190-196.

Marshall PS, Forstot M, Callies A, Peterson PK, Schenck CH (1997). Cognitive slowing and working memory difficulties in chronic fatigue syndrome. Psychosom Med 59: 58-66.

Michiels V, Cluydts R (2001). Neuropsychological functioning in chronic fatigue syndrome: a review. Acta Psychiatr Scand 103: 84-93.

Michiels V, de Gucht V, Cluydts R, Fischler B (1999). Attention and information processing efficiency in patients with chronic fatigue syndrome. J Clin Exp Neuropsychol 21: 709-729.

Owen AM, James M, Leigh PN, Summers BA, Marsden CD, Quinn NP et al (1992). Fronto-striatal cognitive deficits at different stages of Parkinson's disease. Brain 115(Part 6): 1727-1751.

Owen AM, Roberts AC, Polkey CE, Sahakian BJ, Robbins TW (1991). Extra-dimensional versus intra-dimensional set shifting performance following frontal lobe excisions, temporal lobe excisions or amygdalo-hippocampectomy in man. Neuropsychologia 29: 993-1006.

Owen AM, Sahakian BJ, Semple J, Polkey CE, Robbins TW (1995). Visuo-spatial short-term recognition memory and learning after temporal lobe excisions, frontal lobe excisions or amygdalohippocampectomy in man. Neuropsychologia 33: 1-24.
Paul RH, Cohen RA, Gilchrist JM (2002). Ratings of subjective mental fatigue relate to cognitive performance in patients with myasthenia gravis. J Clin Neurosci 9: 243-246.

Reeves WC, Lloyd A, Vernon SD, Klimas N, Jason LA, Bleijenberg $\mathrm{G}$ et al (2003). Identification of ambiguities in the 1994 chronic fatigue syndrome research case definition and recommendations for resolution. BMC Health Serv Res 3: 25.

Reyes M, Nisenbaum R, Hoaglin DC, Unger ER, Emmons C, Randall B et al (2003). Prevalence and incidence of chronic fatigue syndrome in Wichita, Kansas. Arch Intern Med 163: $1530-1536$

Robbins TW (1996). Dissociating executive functions of the prefrontal cortex. Philos Trans $R$ Soc Lond B Biol Sci 351: 1463-1470 discussion 1470-1471.

Robbins TW, James M, Owen AM, Sahakian BJ, Lawrence AD, McInnes L et al (1998). A study of performance on tests from the CANTAB battery sensitive to frontal lobe dysfunction in a large sample of normal volunteers: implications for theories of executive functioning and cognitive aging. J Int Neuropsychol Soc 4: 474-490.

Robbins TW, Sahakian BJ (1994). Computer methods of assessment of cognitive function. In: Copeland JRM, Abou-Saleh MT, Blazers DG (eds). Principles and Practice of Geriatric Psychiatry. John Wiley \& Sons Ltd: Chichester. pp 205-209.

Ross SD, Estok RP, Frame D, Stone LR, Ludensky V, Levine CB (2004). Disability and chronic fatigue syndrome: a focus on function. Arch Intern Med 164: 1098-1107.

Smets EM, Garssen B, Bonke B, De Haes JC (1995). The Multidimensional Fatigue Inventory (MFI) psychometric qualities of an instrument to assess fatigue. J Psychosom Res 39: $315-325$

Swainson R, Robbins TW (2001). Rule-abstraction deficits following a basal ganglia lesion. Neurocase 7: 433-443.

Wagner D, Nisenbaum R, Heim C, Jones JF, Unger ER, Reeves WC (2005). Psychometric properties of the CDC symptom inventory for the assessment of chronic fatigue syndrome. Popul Health Metr 3: 8.

Ware Jr JE, Sherbourne CD (1992). The MOS 36-item short-form health survey (SF-36). I. Conceptual framework and item selection. Med Care 30: 473-483.

Wearden A, Appleby L (1997). Cognitive performance and complaints of cognitive impairment in chronic fatigue syndrome (CFS). Psychol Med 27: 81-90. 